\title{
The Development of Dental Informatics of Oral Cancer Screening for Prevention in Community Level: A Prototype Study in Thailand
}

\author{
Vanvisa Sresumatchai ${ }^{1}$, Boworn Klongnoi ${ }^{2 *}$, Dechavudh Nityasuddhi ${ }^{3}$, Jeeranun \\ Klaewkla ${ }^{1}$, Mongkol Akko ${ }^{1}$
}

\begin{abstract}
Objective: The purpose of this study is to identify general dentists' information needs for oral cancer screening in community and the information sources they use to meet those needs in clinical settings so as to inform the design of dental information systems. Methods: A semi-structured interview was conducted with a convenience sample of 8 general dentists and 5 dental assistant in the Public Health Region 9 area during clinical hours. One hundred and five patient cases were reported by these dentists. Interview transcripts were coded and analyzed using thematic analysis with a constant comparative method to identify categories and themes regarding information needs and information source use patterns. Results: Two top-level categories of information needs were identified: foreground and background information needs. To meet these needs, dentists used four types of information sources: clinical information/tasks, risk factor of oral cancer in general people, surveillance in community and referral to oral cancer treatment. Major themes of dentists' unmet information needs include: (1) timely access to information on various subjects; (2) better visual representations of dental problems; (3) access to patient-specific evidence-based information; and (4) accurate, complete and consistent documentation of patient records. Resource use patterns include: (1) dentists information needs matched information source use; (2) little use of electronic sources took place during treatment; (3) source use depended on the nature and complexity of the dental problems; and (4) dentists and dental nurse routinely practiced cross-referencing to verify patient information. Conclusions: Dentists have various information needs of oral cancer at the point of care. For future development of dental information or clinical decision support systems, developers should consider integrating high-quality, up-to-date clinical evidence into comprehensive and easily accessible EDRs as well as supporting dentists' resource use patterns as identified in the study.
\end{abstract}

Keywords: Dental informatics- dental information- oral cancer screen- design and implementation

Asian Pac J Cancer Prev, 22 (12), 3865-3873

\section{Introduction}

Dental informatics is a field to supporting dental care. Mostly of dentist unknown about the important of dental informatics is, what its meaning are, what it has accomplish and how dentist can get involved in the protocol for screening in community (Schleyer, 2001). Dental informatics can be consider as sub domain of medical informatics that links information technology, communication and healthcare to improve the quality and safety for patient care data. It has involved some part of biomedical informatics and follows by SNODENT standard (Goldberg et al., 2005; Schleyer, 2003). With the overflowing rapid development of computer application and information technology in the past decade, modern dental data research was becoming more dependent on informatics. However, it is important to differentiate informatics from IT.

Informatics focuses on organization or protocol for practice are input and the process that mean the staffs which follow by all of protocol from their organization whilst document reports are output. The informatics focuses on research and evaluation of computing applications, information models, and periodically looks into how did to set up, organize, and process digitization based on existed hardware and software. Because of the information technology is mainly focused on the development and implementation of computer technology and telecommunications. Even though informatics and information technology focus on different aspects of practice and research, they have shared common interests, such as a joint program in which informaticians and information technology engineers collaborate to customize a new three-dimensional (3D) virtual reality 
system for training preclinical dental students. Therefore, dental information technology (d-IT) research could be considered as a subcategory of dental informatics (DI).

In Thailand, Goal 3 is to "Ensure healthy lives and promote wellbeing for all at all ages" from Sustainable Development Goals (SDGs) has been well-established, This presents an opportunity for eHealth to support a comprehensive and coherent approach to health and support integrated, people centered health services. The notion aligns with Thailand 4.0 (Jones and Pimdee, 2017), a policy that is pushing new economic growth and affecting a new way of life in all areas of society. The technology was bringing to support in Thai health system is necessary to set a common guideline for action. For new learning processes create a modern working model on healthcare and are driven quickly. That is a challenging context for Thailand to elevate facilities, fast and accurate service to public health. Thailand 4.0 to Health 4.0 (Estrela et al., 2019) is the essential to integrate eHealth Strategy into a framework of health for moving in the same direction in order to be successful in the long run. The one purpose of eHealth strategy development in Thailand move forward through senior executives and relevance partnership understanding strategic goals of eHealth, which will bring information and communication technology to increase the quality and efficiency of medical and public health services to the maximum benefit of the people (Witoolkollachit, 2017). Singapore leads the report's Networked Readiness Index, followed by Finland, Sweden, Norway and the United States. The10 top five in the region in terms of overall ICT readiness remain China, Malaysia, Mongolia, Sri Lanka, and Thailand, as in 2015. The group of emerging and developing Asian countries has been both moving up and converging since 2012 . Individual usage in the region is still one of the lowest in the world, but has been growing strongly in recent years (Dafny, February 16, 2017).

Oral cancer (ICD C00-C06) satisfies the criteria for a suitable disease for screening1 in several regions of the world. Internationally, oral cancer in ICD $10^{\text {th }}$ edition for coding C00-08 accounted for 300,373 new cancer cases and 145,353 cancer deaths in 2012 (Auluck et al., 2012) while, oral cancer is predominantly diagnosed among males, it can be as common or even more common in females than in males, in many South East Asia: SEA populations (Vatanasapt et al., 2011). All of the 11 countries within the SEA region, Singapore, Thailand, Philippines, Vietnam and Malaysia are the only countries that have population-based cancer registries. The reports from Singapore and Thailand's registries were the most up-to-date with the last report dated 2015, with reporting periods until 2014 and 2012, respectively (Cheong et al., 2017; Virani et al., 2017). From the data on oral cancer specifically, these data were available from Thailand, Philippines and Malaysia registries in the last reporting periods, the latest data for Singapore were not available and data on oral cancer were last reported for 2003-2007 (Cheong et al., 2017). In the north part of Thailand, risk factor exposure was related to gender and age of the patients for the both from tobacco smoking and alcohol consumption were more chewing was frequently observed from males, whereas the betel quid chewing was more in females (Komolmalai et al., 2015). On the 5-year survival rate for oral cancer in most countries is approximately $50 \%$ (Johnson et al., 2011). From the study in some Asian countries indicate survival rates ranging from 38\%-42\% in India and 61\% in Taiwan (Rapidis et al., 2009).

The computer application of bioinformatics is a combination between biology and information technology. The discipline encompasses many computational tools and methods used to manage, analyze, and manipulate large sets of biological data (Fulekar and Sharma, 2008). Form which Hwa A. Lim, bioinformatics is the new subject that is concerned with the collection and analysis of genetic data and its dissemination to the research community. Meanwhile, Dental informatics (DI) was described as "the application of computer and information science to improve dental practice, research, education and management", is a relatively new discipline that has significant potential in supporting the research and education of dental science (Schleyer and Spallek, 2001; Schleyer, 2003). DI can be included a sub-domain of medical informatics (MI), therefore the development of DI to a certain boundary on MI. Regardless of the fact that DI and MI distribute common characteristics in medical research, it is worth having independent investigations focusing on DI itself.

\section{Materials and Methods}

\section{Method}

To develop a dental information system, determine the oral cancer screening in community analysis to be utilized in the performance of prevention oral cancer integrated with information technology. This research was developed in accordance with the System Development Life Cycle - SDLC, using the following three steps. Step one System analysis: developer performed a systems and procedural analysis of the existing system to determine scope of the development. This step provided the development team with important information about the user requirements in this step can two sub-step following areas of system requirements determinations are factfinding and information gathering for analysis of the existing problem to new solve in dental work of oral cancer screen. Requirements structuring for procedural analysis in new system requirement from the existing program in the old system which model chart of new system in the following areas: the structure and form of the dental workflow within the community hospital. Step two System design and development: The results from Step one which consists of sub-systems, were analyzed and used to in the design of the new information system which more accurately met the needs of the target users. The design process involved the following steps in the chart of workflow diagram present about people registrations, examination, diagnosis or additional diagnosis, treatment planning, surveillance, warning message. Context diagram present the top level of data flow diagram about all of the dental information system. The diagram was to present relationship of system to external environment. Dataflow diagram performed communication between the process 
The Development of Dental Informatics of Oral Cancer Screening for Prevention in Community Level

and internal or external environment. The data present about input data for each process and output data from the process of dental information system (oral cancer screen). This process were explain data record, delete data and edit data in the database which call data store for data flow diagram. Entity-relationship diagram or ER-model was the conceptual data modeling present entity relationship. It is tools for overall data simulation of dental information system which identify storage data in database of system. Step three System implementation: it was deployed for trial and evaluation as preparation with the application guide, system installation after that user questionnaire to evaluate satisfaction analysis and preparation of summary report. This research study involves the experimental development of a prototype oral cancer screen in dental information system. Judgment sampling was used to select the study subjects of dentists and dental assistant staff in community hospital. The sample consisted of 59 general dentists and 15 dental assistants system stakeholders considered representative of oral cancer screen in hospital community. The oral cancer screen web base application was installed at specific workstations associated with oral cancer screen within the Public Health Region area 9 in Northeast of Thailand. This study was approved by the Research Ethics Committee, Mahidol University.

\section{Protocol of oral cancer screen}

We applied an inductive analysis approach, grounded in the respondents' answers without any preconceived assumptions on the part of the researchers. Specifically, we used the thematic analysis method to analyze the interview transcripts. Thematic analysis is a method for identifying, analyzing and reporting patterns (themes) in oral cancer data and process for screening. In contrast to other qualitative methods, such as grounded theory or narrative analysis, thematic analysis is not wedded to any pre-existing theoretical framework; thus it can be used within different theoretical frameworks as either a realist or constructionist method.

We conducted the analysis following the methods of Braun and Clarke (Braun and Clarke, 2006). Two trained analysts open-coded the transcripts independently to identify categories regarding dentists' information needs and use of information sources, with special attention to their unmet information needs. They then compared the codes and reached consensus on coding discrepancies through discussion. Any code identified was matched with data extracts that demonstrated that code. All data extracts were then collated together within each code. One researcher then related data extracts within and across categories to identify potential themes, and the whole team systematically reviewed these themes to make sure that they worked in relation to both the coded extracts and the entire data set.

\section{Dental information design}

We analyzed the three categories of concerns and used the results to guide design of the new system in three steps, logical design, user interface design, and database design. The logical design of a system involves planning and mapping data flow and can be represented as context diagrams. For our system the data flow was mapped through the people enrollment process, the treatment phase and the reporting phase. People/Patient registration process: In the first step involves entry of provider information, people/ patient demographic data, risk factor of oral cancer and village health volunteer had to take care the people in village. The Dental Information System people/patient records are searchable from the patient's hospital number, first name, last name, identification (ID) number, and gender. The system records new entered data, edited information and clinical status updates which are automatically saved to the patient system. Oral cancer screening processes: in this process consists of five sub-processes: oral cancer screen by village health volunteer (S1), dental assistant (S2), dentists (S3), surveillance and treatment monitoring. Diagnosis process occurs in the examination room as the dentist performs the physical exam, takes the patient's history and reviews diagnostic tests. In this part of the process the patient's record is updated with data about disease history, changes in the dental or medical history and the review of current symptoms. The dental history of risk group contains data related to history of dental records is related to smoking, chewing tobacco or snuffing, alcohol consumption, betel quid consumption, long durations of sun exposure while working, denture wear for $>1$ year, irritation of oral mucosa, and/or history of head and neck cancer. The treatment monitoring process involves follow-up care of the patient after the initial treatment intervention. Follow-up data collected in this process includes interval history and examination, surgical procedure follow-up, and monitoring of treatment side effects.

\section{Protocol of oral cancer screen}

Input can be divided into three levels in the process of screening for oral cancer. Level 1 village health volunteer(VHV) screening incorporates factors such as smoking/used to smoke, snuff (dry nasal tobacco)/ tobacco/used, chewing tobacco/chewing betel nuts/used, consuming alcohol, working in the sun on a regular basis, dentures for more than one year, and mouth ulcer. The questionnaire results from S1 will be used for level 2 dental nurse screening for risk people groups. In a community hospital (level 3), dentistry conduct oral examinations on people who have lesions, and the output covers the amount of oral screening and treatment from 1 to 3 as well as all of the surveillance questionnaires from each level.

\section{Web based system architecture of oral cancer screening}

For import patient database registry and management, a customized software module designed by the oral cancer screening program was added to the existing Health Information System (HIS) program from Public Health Region 9 data center. The web based application allowed data entry and retrieval regarding the risk factors recorded from S1 and S2 levels, characteristics of the lesions found in S2 and S3 levels, and the treatment received by each patient at the S3 level. Even at the most practical level, DSN stands for Domain Name System. DNS performs an essential lookup from a domain name for "ocphmodel. 
dt.mahidol.ac.th" to a Mahidol University IP address, which is usually required for a computer to route a request to the appropriate server. Before going into the specifics of loading the HIS database, their dental required to take a step back as well as export data from the "OralCancer Maharat Model" to an existing OCPH system. The HIS database receives data from Load HIS, which includes information such as name, age, address, village, and village health volunteer (VHV) name. The oral cancer screen level 1(S1) through oral cancer screen level 1(S3) and oral potentially malignant disorder (OPMD) lesions surveillance for the part of screening, treatment, and follow up to use at hospital for patients with oral cancer would be included in the web-based application server for data entry about oral cancer screening online. According to my research, web-based applications include more databases to store dental data. Databases allow you to define dental data structures, contribute new data, query for existing data, updating and deleting existing data, computation across the data, and more. SQL databases, which stand for "Structured Query Language," keep data in tables that are linked together by common IDs, which are usually integers. A caching service is a simple key/value dental data store that allows you to save and retrieve data in less than $\mathrm{O}(1)$ time. Applications often use caching services to save the results of computations for oral cancer screening so that they can be retrieved from the cache rather than recomputing them the next time for dentistry and dental staffs. Job queues store a list of jobs that need to be run asynchronously.

\section{Dental information design}

The Iterative and Incremental Development or SDLC will be utilized in five steps to produce dental information design for screening and monitoring oral cancer patients: planning, analysis, design, development, installation, and maintenance. Analysis for developing an information system for monitoring oral cancer risk groups is divided into two parts as follows: The first part present in Circle No. 1 was developed in an interactive manner that developed the oral cancer screening system. For the first year of the study effort, a pilot version used an on-site trial to examine data recording formats from primary, secondary, and tertiary screening. The pilot version focuses on the oral cancer screening and testing paradigm at the S1-S3 levels. The outer ring (number 2 ) in the second part will analyze the system with the connection point from the outer loop (as shown by the red arrow), and there are three steps that must be looped, repeated, and added, namely analysis (Analysis), design (Design), development (Development), and installation (Implementation) by starting the development of an information system to monitor oral cancer risk groups of the first cycle. After the implementation of the system development planning phase, the research team makes a plan, the number of iterations to and the system to be developed. This is done in the iterative and repetition of the system in the process of system analysis, design, development and installation. It will repeat the analysis point that appears an arrow dragged from the outer loop is SDLC connected to the position of the analysis in the inner circle is an interactive system development. The community-level monitoring method for oral cancer is an analysis of the initial system, and it was discovered that it did not address surveillance for risk individuals groups in the community. System analysis and design for dental informatics on the deployment of oral cancer screening on the screening and warning system are included. Warning system, there are three levels of warning for risk groups to get oral cancer screening. Level 1 st by sending warning messages to mobile phones of risk groups and family caregivers to participate in the oral cancer risk survey with VHV. In the case of risk groups failing to be evaluated at Tambon Health Promoting Hospital, the level 2nd system will send a reminder to attend an oral cancer screening with a dental nurse. The system has repeatedly alerted at the ending of level 3 for risk groups should have been examined for oral cancer at Tambon Health Promoting Hospital. In the event that there is a lesion of either PMD or oral cancer, dentists will be referred for treatment in the patient's treatment rights system, as well as establishing a network of doctors and dentists specializing in oral cancer. In the situation that the hospital still does not have a specialized medical team to conduct treatment, an e-referral method was designed to refer patients to other hospitals.

\section{Results}

\section{Demographics}

Eight dentists and five dental assistant completed the study ( 5 males and 8 females). They were on average 45 years old (range: $30-55$ years) and had been practicing for 20.7 years (range: 2-32 years). Eight of general dentists and five dental assistant used sometime an oral cancer screening practice management system for patient scheduling and welfare scheme.

\section{Information needs of general dentists}

Dentists' information needs for oral cancer screening varied by nature and number depending on the oral cancer screening options and patient characteristics. For the 105 patient cases, dentists reported a total of 320 information needs (256 met needs and 64 unmet needs), averaging 3.1 per patient (range: 1 to 7). Two top-level categories of information needs with several subcategories emerged from the coding: background information needs and foreground information needs.

\section{Background information needs}

Dentists also inquired about issues beyond the medical or biological aspects of a dental problem. These information needs, such as population at risk, dental health behaviors and welfare scheme, though at the background of the current problem, are indispensable in helping dentists to reason about possible causes of the problem to make well-informed diagnoses and joint treatment decisions with patients. 
The Development of Dental Informatics of Oral Cancer Screening for Prevention in Community Level Table 1. Information Needs of General Dentists during Oral Cancer Screening in the Study $(\mathrm{n}=105)$ Patient Cases

\begin{tabular}{|c|c|c|c|c|c|c|c|}
\hline $\begin{array}{l}\text { Background } \\
\text { information needs }\end{array}$ & $\begin{array}{l}\text { Total } \\
\text { case }\end{array}$ & $\begin{array}{l}\text { Cases with } \\
\text { needs }\end{array}$ & $\begin{array}{c}\text { Cases with } \\
\text { needs unmet }\end{array}$ & Foreground information needs & $\begin{array}{l}\text { Total } \\
\text { case }\end{array}$ & $\begin{array}{l}\text { Cases with } \\
\text { needs }\end{array}$ & $\begin{array}{l}\text { Cases with } \\
\text { needs unmet }\end{array}$ \\
\hline $\begin{array}{l}\text { Population at risk for oral } \\
\text { cancer }\end{array}$ & 36 & $33(92.3) \%$ & $3(8.10 \%)$ & Chief complaint \& symptoms & 55 & $55(100 \%)$ & $0(0 \%)$ \\
\hline Welfare Scheme & 17 & $12(71.40 \%)$ & $5(29.20 \%)$ & $\begin{array}{l}\text { Oral cancer screening options \& } \\
\text { procedures }\end{array}$ & 50 & $44(88.20 \%)$ & $6(12.50 \%)$ \\
\hline Service plan & 11 & $11(100 \%)$ & $0(0 \%)$ & Visual representation of problems & 43 & $31(72.30 \%)$ & $12(28.10 \%)$ \\
\hline Referral for treatment & 8 & $8(100 \%)$ & $0(0 \%)$ & Surveillance & 41 & $36(88.10 \%)$ & $5(12.40 \%)$ \\
\hline Dental health behavior & 8 & $7(87.50 \%)$ & $1(22.50 \%)$ & Effect of oral cancer screening & 15 & $12(80.10 \%)$ & $3(20.20 \%)$ \\
\hline Access to resources & 8 & $4(50.20 \%)$ & $4(50.10 \%)$ & Warning for next follow-up & 9 & $9(100 \%)$ & $0(0 \%)$ \\
\hline
\end{tabular}

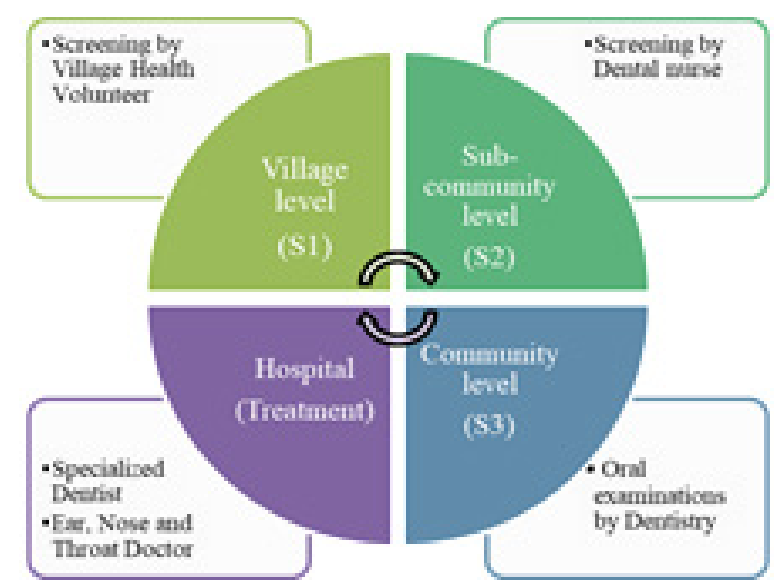

Figure 1. Oral Cancer Screening Framework

\section{Foreground information needs}

Information needs in this category refer to the questions or concerns a dentist had regarding the medical or technical for oral cancer screening aspects of the current dental disease or condition at hand, such as the symptoms, treatment options/procedures, effect of treatment and warning for next follow-up.

\section{Database Design}

PhpMyAdmin software was used to create the database which was named "OCPH.mdb" and consisted of a series of data tables organized in relational database. The results of the oral cancer screening route were used to construct an information system called "DICOCS" (Dental Informatics for Oral Cancer and Surveillance). The design of the information storage system for oral cancer screening in

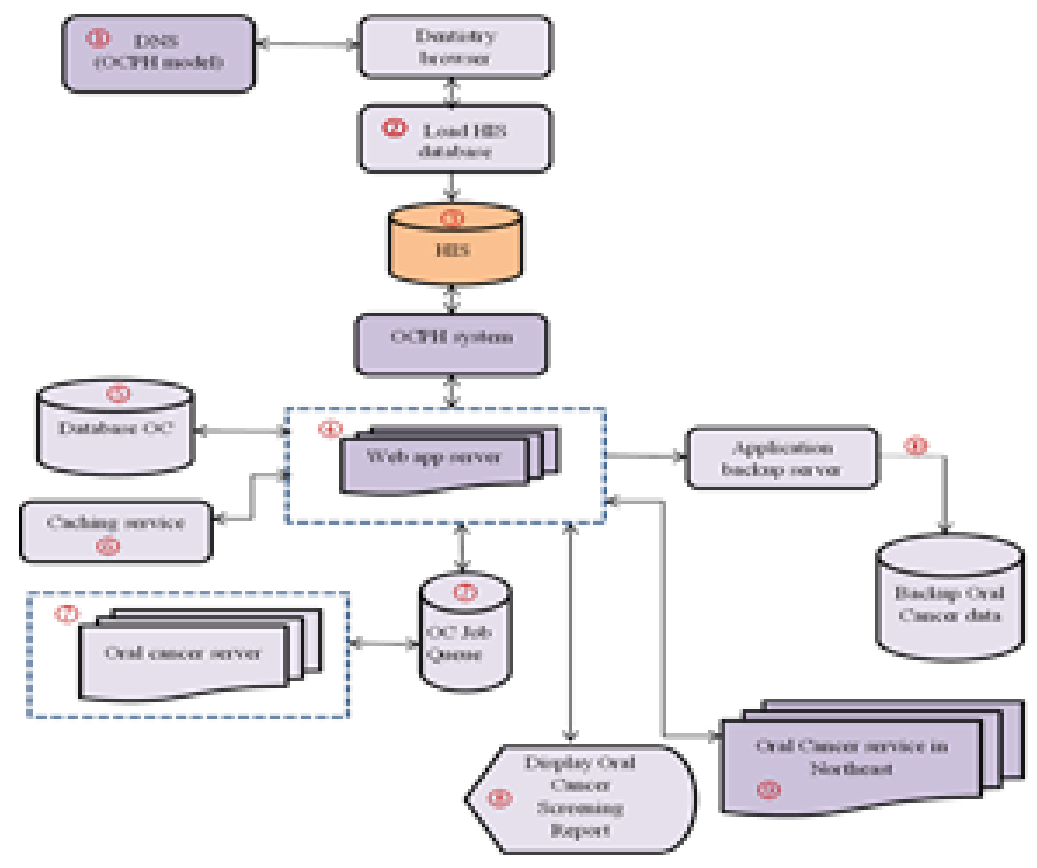

Figure 2. System Architecture of Oral Cancer Screening 
Table 2. Evaluation of Satisfaction with the Dental Information Program (Oral Cancer Screen)

\begin{tabular}{|c|c|c|c|c|c|}
\hline & Participant & $\mathrm{N}$ & Mean & SD. & Std. Error Mean \\
\hline The development of agility & Dentistry & 8 & 4.25 & 0.463 & 0.164 \\
\hline program & Dental Assistant & 2 & 4 & 0 & 0 \\
\hline The development of automated & Dentistry & 8 & 4.25 & 0.463 & 0.164 \\
\hline \multicolumn{6}{|l|}{ management features response } \\
\hline to requirements of users & Dental Assistant & 2 & 4 & 0 & 0 \\
\hline Data entry is convenient & Dentistry & 8 & 4.63 & 0.518 & 0.183 \\
\hline and easy to verify & Dental Assistant & 2 & 4 & 0 & 0 \\
\hline \multirow[t]{2}{*}{ Manuals to understand the overall system and lead to a subsystem } & Dentistry & 8 & 4.13 & 0.641 & 0.227 \\
\hline & Dental Assistant & 2 & 5 & 0 & 0 \\
\hline Manuals to identify functionality & Dentistry & 8 & 4.13 & 0.991 & 0.35 \\
\hline of the system & Dental Assistant & 2 & 4.5 & 0.707 & 0.5 \\
\hline The system can & Dentistry & 8 & 4.25 & 0.463 & 0.164 \\
\hline fast search accurate & Dental Assistant & 2 & 4.5 & 0.707 & 0.5 \\
\hline \multirow[t]{2}{*}{ The system can fast search accurate } & Dentistry & 8 & 4.25 & 0.463 & 0.164 \\
\hline & Dental Assistant & 2 & 5 & 0 & 0 \\
\hline Occupation System can be & Dentistry & 8 & 4.25 & 0.463 & 0.164 \\
\hline Storage data completely & Dental Assistant & 2 & 5 & 0 & 0 \\
\hline \multirow[t]{2}{*}{ System can be reduce the working time of personnel } & Dentistry & 8 & 4.25 & 0.707 & 0.25 \\
\hline & Dental Assistant & 2 & 5 & 0 & 0 \\
\hline \multirow{2}{*}{$\begin{array}{l}\text { Systems allows reduce errors in } \\
\text { the operation associated with data }\end{array}$} & Dentistry & 8 & 4.63 & 0.518 & 0.183 \\
\hline & Dental Assistant & 2 & 5 & 0 & 0 \\
\hline \multirow{2}{*}{$\begin{array}{l}\text { System allows the preparation of a report presented to the dentist } \\
\text { /Managers are rapid }\end{array}$} & Dentistry & 8 & 4.63 & 0.518 & 0.183 \\
\hline & Dental Assistant & 2 & 4.5 & 0.707 & 0.5 \\
\hline Printing system reports less than & Dentistry & 8 & 4.63 & 0.518 & 0.183 \\
\hline the original & Dental Assistant & 2 & 4.5 & 0.707 & 0.5 \\
\hline \multirow[t]{2}{*}{ The system has useful for patients } & Dentistry & 8 & 4.63 & 0.518 & 0.183 \\
\hline & Dental Assistant & 2 & 4.5 & 0.707 & 0.5 \\
\hline \multirow[t]{2}{*}{ You were satisfied to use the system } & Dentistry & 8 & 4.63 & 0.518 & 0.183 \\
\hline & Dental Assistant & 2 & 4.5 & 0.707 & 0.5 \\
\hline
\end{tabular}

a network model, in which DICOCS is the information center and has a hospital as a research area to record the screening information through an information system. Design of a storage system and an information system in network format Where DICOCS is the information center and a hospital serves as a client to capture screening data via an information system.

\section{System Implementation and User Interface}

This OCPH is implemented utilizing a web-based

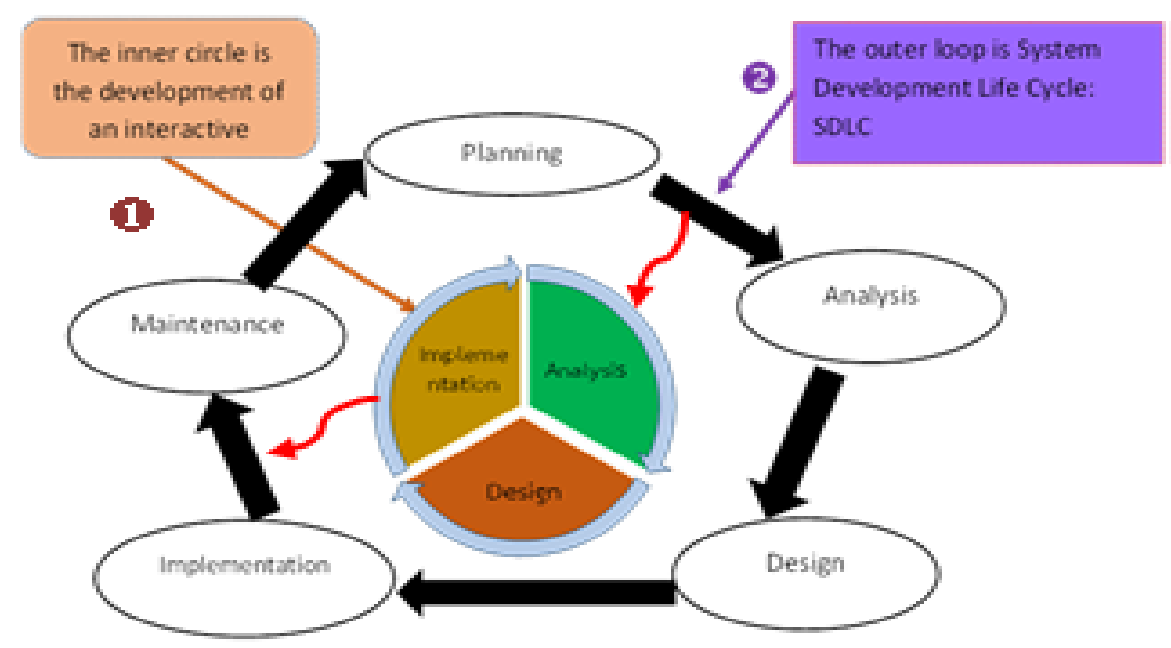

Figure 3. The SDLC Design in Risk Groups for Screening and Monitoring of Oral Cancer 


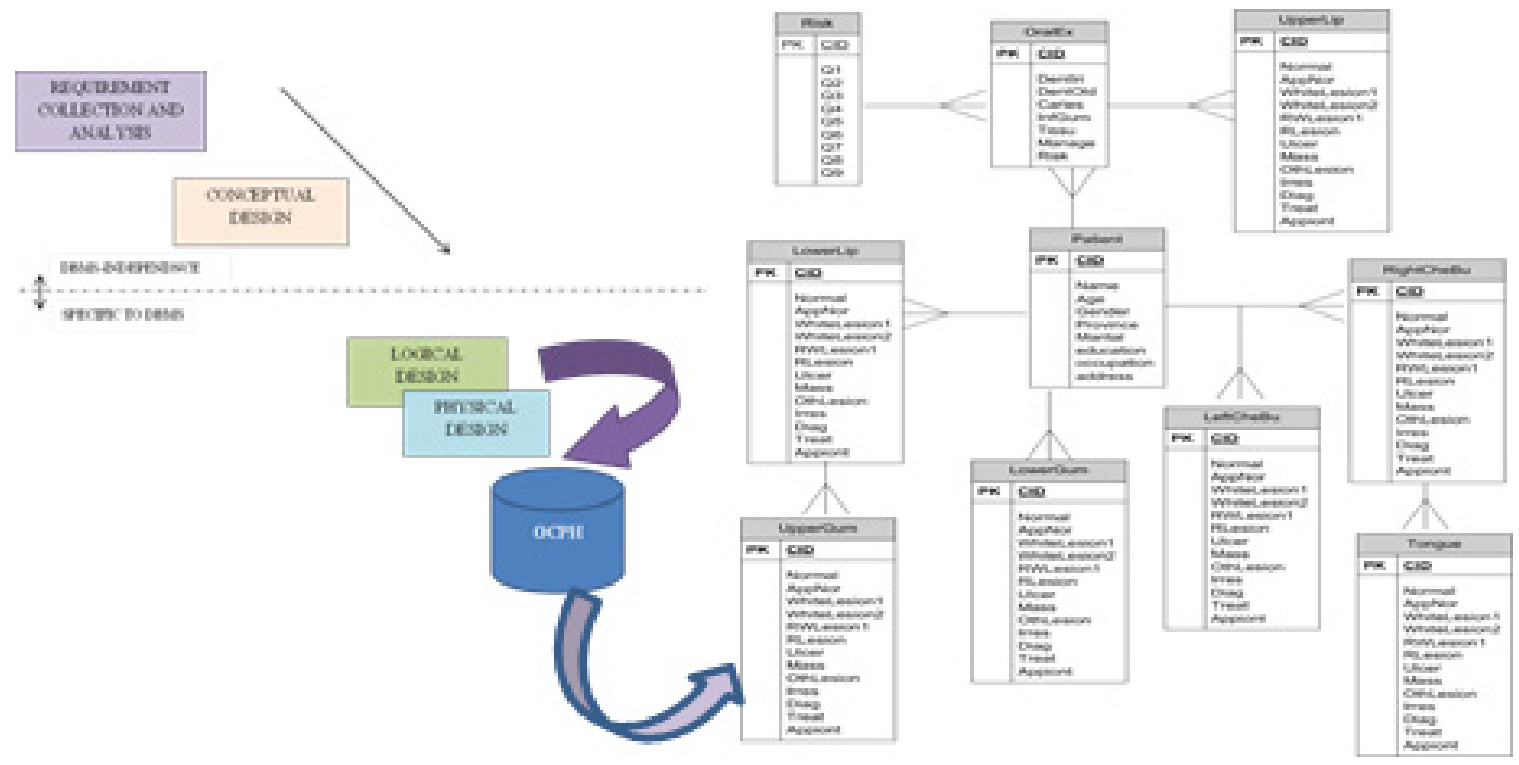

Figure 4. Schematic Diagram of the OCPH Building Design

application, which is the first dental informatics system, with MySQL as the primary database management system. The database connectivity has been done with PHP and Visual Basic.NET as a third-generation programming language. The Visual Basic language supports rapid application development (RAD) and graphical user interface (GUI), database access, and more running under the .NET Framework. The study included a target group comprising people from four provinces: the Nakhon Ratchasima province $(203,685$ participants, 177,371 (87.08\%) underwent oral cancer screening), the Chaiyaphum province (105,523 participants, 99,155 (94.90\%) underwent oral cancer screening), the Buri Ram province $(56,946$ participants, $85.98 \%$ underwent oral cancer screening), and the Surin province $(26,242$ participants, $87.07 \%$ underwent oral cancer screening). The system helped dentists save time when performing oral cancer screening in rural areas and obtaining oral cancer screening data, including monitoring and followup data after oral cancer treatment. In this paper, our team proposed a dental informatics system for use in community oral cancer screening by VHVs, dental nurses, and dentists. We used oral cancer screening information gathered from the home page of OCPHMODEL, which is hosted by Mahidol University, to populate our dental database and simulate the system. Dental specialists use the oral cancer screen information page for the information needed to conduct community surveillance. This proactive dental screening in rural communities detected oral cancer lesions in their early stages.

\section{System Evaluation}

When the development of the application was completed it was web base application and installed on "DICOCS" workstations for trial simulation in the community hospital of Public Health Region Area 9. It was intended that the software be tested on the target population of dentists and dental assistants whose input guided the software development process.

\section{Discussion}

Dentists in the study reported a variety of information needs, with inquiries on oral cancer screening protocol, chief complaints and symptoms, treatment options and procedures and parameters of problems at the top of the list. Regarding medical issues, dentists were most concerned about the impact of patients' pre-existing medical and health conditions (e.g., hypertension and diabetes) on their dental problems, especially during on eight type of oral potentially malignant disorders; OPMDs) such as leukoplakia, erythroplakia, palatal lesions in reverse smoker, oral submucous fibrosis, lichen planus, discoid lupus erythematosus, actinic cheilitis, and inherited cancer syndromes . It appears that the rising prevalence of chronic diseases challenges general dentists in their understanding of oral cancer interactions and the impact of multiple drug regimens. The knowledge of these information needs can inform the design of a dental information system, such as an electronic dental record (EDR). For example, in this study, dentists frequently searched for information on oral cancer screening or previous treatments and procedures, such as the clinical description of leukoplakia or lichen planus in the progress notes section of the patient records. Therefore, progress notes could be highlighted and placed in a more prominent position in the layout of the EDR so that during a subsequent visit dentists can access previous treatment information on the first screen without having to search for them.

In this study, some dentists desired more evidence based information. A few dentists were trying to adopt an Evidence-Based Dentistry (EBD) treatment approach, but felt insufficient support due to the lack of evidence based information accessible at the point of care, such as clinical practice guidelines of oral cancer screening, proposed new classification of oral potentially malignant disorders. This is supported by the Hannes and colleagues' study, which reported a lack of up-to-date evidence for devices and products and the complexity of guidelines regarding treatment choices as two major barriers to an 
EBD approach (Hannes et al., 2008). While information on The Cochrane Collaboration's Oral Health Group's website http://www.ohg.cochrane.Org/reviews.html lists 91 published systematic reviews of dental treatments and interventions as of August 2009, there are only three clinical recommendations on the ADA's EBD Website http://ebd.ada.org. Therefore, one focus of dental information systems could be on designing tools that help search for the most up-to-date clinical evidence and guidelines that match patient characteristics and better integrate this information into dental records in userfriendly way.

Getting quicker access to information, especially for the policy maker should be for service plan for oral cancer screening, has also become a pressing need for dentists. Examples in this study show that patient care was sometimes unnecessarily delayed by the slow preauthorization process required by service plain. Policies that include oral cancer screening into service plain of cancer screening help reduce risk for oral cancer in community and would greatly benefit patients by speeding up treatment.

They needed more evidence-based information, yet some had suspicions about the validity of research studies; they wanted to completely switch over to electronic patient records, yet harbored serious doubts about the authenticity and safety of computer data; they called for more cooperation and compliance from patients, yet sometimes did not fully trust what the patients said.

\section{Limitations}

This study has several limitations. First, we interviewed a convenience sample of dentists in Public Health Region 9 area in Thailand who volunteered to participate in the study. They might be systematically different from dentists who did not choose to participate in terms of information needs and source use. As a result, possible selection bias may exist, with this having an unknown impact on the study results. Therefore, caution needs to be taken when generalizing the results to other general dentists. Second, previous research has suggested that the office interview method might incur potential biases in two ways. On one hand, the interview may stimulate the dentists to think of more questions for our interviewer that were not there if they were not asked (Barrie and Ward,1997), on the other hand, dentists and dental nurse may report fewer information needs for oral cancer screening than they normally would due to their reluctance to reveal ignorance in front of the interviewer (Gerrity et al., 1990). However, we are less concerned about the first bias as raising new questions about a patient in retrospection can still be helpful as long as they are important and relevant oral cancer screening to the patient's diagnosis and treatment. Third, given the fast-paced schedule of dentists, it is difficult to conduct in-depth interviews in dental hospital offices; while we tried to follow up with the most crucial questions on the dentists' information needs and source use, time was not sufficient for the dentists and dental nurse to elaborate more on some of their answers.

In conclusion, a challenge for designing useful oral cancer dental information systems in dentistry is to incorporate evidence-based information based on dentists' information needs and integrate the system seamlessly into the complex dental workflow. As a critical step, we need to identify population at risk for oral cancer screening information needs and source use patterns. This study reveals that general dentists need a variety of information at the point of care, encompassing the medical aspects of dental problems and other issues affecting the condition in oral cancer screening. For optimal patient outcomes, quicker access to comprehensive patient at risk oral cancer and welfare scheme information, better visual representation of patient problems, and access to key evidence-based guidelines of oral cancer treatment are required. These findings can be used to inform the design of dental informatics tools. For example, the development of a comprehensive but easily accessible electronic dental record integrated with high-quality, up-to-date clinical evidence and 2D or 3D visual representation capabilities is a promising direction and guideline for oral cancer screening include image book for eight type of oral potentially malignant disorders .

This study also demonstrates the usefulness of qualitative methods in providing an in-depth understanding of the issues and challenges related to dentists' information needs and source use in clinical settings. Similar techniques can be used to investigate information needs of other members of the profession such as dental specialists and hygienists.

\section{Author Contribution Statement}

Study design: Vanvisa Sresumatchai, Boworn Klongnoi. Data collection: Vanvisa Sresumatchai. Data analysis and interpretation: Vanvisa Sresumatchai, Boworn Klongnoi ,Dechavudh Nityasuddhi,Jeeranun Klaewkla,Mongkol Akko. Discussion of the results: Vanvisa Sresumatchai, Boworn Klongnoi. Writing and/ or critical review of the content: Vanvisa Sresumatchai, Boworn Klongnoi, Dechavudh Nityasuddhi, Jeeranun Klaewkla. All authors approved the final version.

\section{Acknowledgements}

Our research team would like to thank the dentists, dental nurses, and village health volunteers of Health Area 9, Nakornchai Burin for their valuable help in this work.

\section{Funding statement}

Vanvisa Sresumatchai received funded by the government budget grant from Mahidol University, Thailand - funding code RES15455.

\section{Origin of the article}

This article was extracted from the research project entitled "Development of Dental Information Center in Oral Cancer Surveillance on Maharat Model and Health Literacy of Oral Cancer Prevention".

\section{Ethical approval}

Ethical approval for the study was obtained from the Research Ethics Committee of Faculty of Dentistry and 
The Development of Dental Informatics of Oral Cancer Screening for Prevention in Community Level

Faculty of Pharmacy, Mahidol University, Thailand (COA MU-DT/PY-IRB 2019/041.0307).

\section{Data availability statement}

All data generated or analyzed during this study are in the custody of the researchers and will be made available upon request.

\section{Statement of conflict of Interest}

There is no conflict of interests.

\section{References}

Auluck A, Hislop T, Bajdik C, et al(2012). Gender- and ethnicityspecific survival trends of oral cavity and oropharyngeal cancers in British Columbia. Cancer causes Control, 23, doi:10.1007/s10552-012-0065-0.

Barrie AR, Ward AM (1997). Questioning behavior in general practice: a pragmatic study. $B M J, \mathbf{3 1 5}, 1512-5$

Braun V, Clarke V (2006).Using thematic analysis in psychology. Qual Res Psychol, 3, 77-101

Cheong S, Vatanasapt P, Yang,Y-H, et al (2017). Oral cancer in South East Asia: Current status and future directions. Transl Res Oral Oncol, 2, 2057178X1770292.

Dafny L (2017). New Marketplace Survey: The Sources of Health Care Innovation. Retrieved from Harvard Business School.

Estrela V, Monteiro A, França R, Abdeldjalil K, et al (2019). Health 4.0: Applications, Management, Technologies and Review. 4. doi:10.26415/2572-004X-vol2iss1,262-276.

Fulekar M, Sharma J (2008). Bioinformatics applied in bioremediation. Innov. Roma Food Biotechnol, 2.

Goldberg L, Ceusters W, Eisner J, Smith B (2005). The significance of SNODENT. Stud Health Technol Inform, 116, 737-742.

Gerrity MS, DeVellis RF, Earp JA (1990). Physicians' reactions to uncertainty in patient care. A new measure and new insights. Med Care, 28, 724-36.

Hannes K, Norre D, Goedhuys J, Naert I, Aertgeerts B (2008). Obstacles to implementing evidence-based dentistry: a focus group-based study. J Dent Educ, 72, 736-44.

Johnson NW, Jayasekara P, Amarasinghe AA (2011). Squamous cell carcinoma and precursor lesions of the oral cavity: epidemiology and aetiology. Periodontol, 57, 19-37.

Jones C, Pimdee P (2017). Innovative ideas: Thailand 4.0 and the fourth industrial revolution. Asian Int J Soc Sci, 17, 4-35.

Komolmalai N, Chuachamsai S, Tantiwipawin S, et al (2015). Ten-year analysis of oral cancer focusing on young people in Northern Thailand. J Oral Sci, 57, 327-34.

Rapidis AD, Gullane P, Langdon JD, et al (2009). Major advances in the knowledge and understanding of the epidemiology, aetiopathogenesis, diagnosis, management and prognosis of oral cancer. Oral Oncol, 45, 299-300.

Schleyer TSH (2001). Dental informatics: A cornerstone of dental practice. J Am Dent Assoc, 132, 605-13.

Schleyer TKL (2003). Dental Informatics: An Emerging Biomedical Informatics Discipline, 67, 1193-1200.

Vatanasapt P, Suwanrungruang K, Kamsa-Ard S, Promthet S, Parkin MD (2011). Epidemiology of oral and pharyngeal cancers in Khon Kaen, Thailand: a high incidence in females. Asian Pac J Cancer Prev, 12, 2505-8.

Virani S, Bilheem S, Chansaard W, et al (2017). National and subnational population-based incidence of cancer in Thailand: Assessing Cancers with the Highest Burdens. Cancers, 9, 108.

Witoolkollachit P (2017). eHealth Strategy, Ministry of Public
Health (2017- 2026). Retrieved from Thailand: https:// ehealth.moph.go.th.

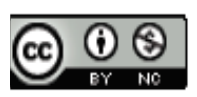

This work is licensed under a Creative Commons AttributionNon Commercial 4.0 International License. 